\title{
Interfaces amigables para la recuperación de la información bibliográfica
}

\author{
Francisco Javier García Marco \\ Universidad de Zaragoza \\ E-mail: jgarcia@cc.unizar.es
}

\subsection{Resumen}

Se analiza el concepto de interfaz amigable y la función clave que ocupa en los sistemas automatizados de información: el control de su interacción con el operador humano. Se propone una definición operativa de amigabilidad, que permita la evaluación eficaz del interfaz. Se traza una breve historia de su desarrollo, sus fundamentos cognitivos y las técnicas más comunes utilizadas para facilitar la comunicación entre el hombre y los sistemas automatizados. Los conceptos discutidos son ejemplificados mediante la descripción de tres prototipos innovadores en el campo de la información bibliográfica, cuya eficacia ha sido suficientemente contrastada y documentada: Icarus, The Book House y Okapi. Finalmente, se presenta una panorámica prospectiva de los desarrollos futuros que cabe predecir en el campo de la interacción entre los sistemas bibliográficos y los usuarios.

Palabras clave: Interfaces amigables. Información bibliográfica. OPAC. Recuperación de información. Interacción hombre/máquina.

\subsection{Abstract}

The concept of friendly interface is analysed together with the key position it occupies in information retrieval systems: the control of interaction with the human operator, and the optimisation of the relationship between them. An operative definition of friendliness is proposed, to ensure the proper evaluation of an interface. A schematic history of the human-machine interface development is traced, and its cognitive backgrounds and development techniques are considered. The theoretical concepts explored are exemplified with the description of three innovative prototypes in the field of bibliographic information, whose effectiveness has been properly contrasted and documented: Icarus, The Book House and Okapi. Finally, a prospective landscape of the foreseeable future developments in the field of interaction between bibliographic information sys-

Scire. $1: 1$ (en.-jun. 1995). 
tems and users is presented.

Keywords: Friendly interfaces. Bibliographic information. OPAC. Information Retrieval. Human-machine interaction.

\section{Conceptos previos}

El tema de los interfaces hombre-máquina se han configurado como una de las áreas de investigación críticas para el desarrollo de la sociedad de la información. No en vano, el interfaz de usuario regula la interacción entre ambos elementos del sistema, y en virtud de ello se convierte en un eslabón especialmente delicado. Así, por ejemplo, ante dos programas semejantes en su funcionalidad formal, la amigabilidad del interfaz — es decir, su potencial de generalización por el usuario- determina directamente el mercado potencial de cada uno de los producto. La amigabilidad condiciona si el programa se va a dirigir a un sector especilizado o si podrá alcanzar el mercado de masas, y, en virtud de su potencial medio de generalización en el aprendizaje, condiciona en manera importante su velocidad de penetración en el mercado.

Así pues, los interfaces amigables son un área de investigación de creciente importancia, conforme las organizaciones que ofrecen productos y servicios de información pretenden conquistar un sector mayor de consumidores. Sin embargo, se trata también de un área en la que existe considerable confusión. Por ello, vamos, antes de nada, a dedicar algunas palabras a presentar los conceptos fundamentales que componen el título del trabajo: discutiremos el concepto de recuperación de información y definiremos lo que cabe entender por interfaz y por el más escurridizo término de amigabilidad.

El concepto de recuperación de información debe ser interpretado dentro del paradigma clásico de la recuperación de información tal y como ha quedado elaborado por la comunidad científica implicada en la construcción de la Information Science. Dicho modelo es bien conocido: una determinada necesidad de información es formulada (simbolizada) en una pregunta; esa pregunta es traducida a un lenguaje entendible por el sistema de almacenamiento, conservación y recuperación de información (SACRI), y esa pregunta, una vez reformulada en términos del SACRI, produce una respuesta, que cabe evaluar en términos de ruido y llamada (1).

Como todos los autores que tratan el tema se han ocupado de resaltar, este paradigma revela un proceso esencialmente comunicativo y de carácter complejo, es decir, compuesto de varias fases y con implicación de, muchas veces, numerosos intermediarios. Como en cualquier fenómeno comunicativo, el resultado final está amenazado por factores de muy diversa índole: el usuario puede fracasar en el intento de simbolización de su necesidad; la pregunta puede ser

Scire. 1 : 1 (en.-jun. 1995). 
malinterpretada, etc. En este contexto comunicativo los problemas derivados del uso del código adquieren una especial importancia, dado que, entre la necesidad originaria y la respuesta final, se producen varias operaciones de traducción o recodificación. El éxito de estas operaciones está amenazado tanto por fenómenos léxicos - la sinonimia, la homonimia, la polisemia y la pertenencia léxicacomo sintácticos - la versatilidad de la estructura superficial de los lenguajes naturales, que genera problemas análogos a los anteriores pero a nivel meta-léxico- Dichos problemas se extreman en ausencia de mediadores capacitados (documentalistas, bibliotecarios referencialistas, etc.), y, especialmente, en entornos automatizados.

La forma de luchar contra esa ambigüedad léxica es precisamente simplificar y asegurar la biunivocidad entre los términos y operadores de la pregunta, por un lado; y los que se encuentran almacenados en las representaciones documentales del SACRI y su lenguaje de recuperación, por el otro. Los resultados de estas operaciones de control lingüístico son, por una parte, los índices de palabras clave -más o menos organizados a priori o a posteriori en auténticos lenguajes de descripción documental —, y, por la otra, los lenguajes de interrogación. Este control y normalización lingüística es extremadamente eficaz; pero, desgraciadamente, se aleja enormemente de los usos comunicacionales de gran parte de los usuarios potenciales de nuestros servicios de información.

Precisamente, es a la parte del SACRI que encargada de interactuar con el usuario a la que se denomina interfaz de usuario. También podemos definirla, en relación con los problemas antes señalados, como la parte de un SACRI que controla la comunicación con el usuario (2). Así por ejemplo, en una biblioteca el interfaz de usuario lo compone la parte pública del OPAC, pero también el tiempo y cualidades dedicadas a los usuarios por parte del personal bibliotecario. En este trabajo, sin embargo, y sin menospreciar ( $;$ antes al contrario!) la importancia del interfaz humano, nos ocuparemos tan sólo del interfaz hombre/máquina. En cualquier caso, y de forma global, las características del interfaz de usuario constituyen uno de los factores determinantes del acceso a la información, tan importante en último término como la misma disponibilidad física (almacenamiento) de esa información.

La amigabilidad o cordialidad de un interfaz de usuario se refiere precisamente a su facilidad de uso. Borenstein (1991) lo ha expresado de forma sencilla, con la afirmación de que implicarse en la creación de interfaces amigables es, lisa y llanamente, programar "como si la gente realmente importase". Esa facilidad de uso es, por supuesto, relativa al tipo de usuario; pero de una manera general podemos decir que un interfaz es tanto más amigable cuanto más fácil de usar resulta para una mayor proporción de usuarios de una población dada. Esta facilidad de uso está muy relacionada con otro concepto al que no necesariamente se

Scire. 1 : 1 (en.-jun. 1995). 
asimila, a saber, con el de interactividad. Un interface es interactivo si "dialoga" con el usuario, si le proporciona feedback comunicativo. Esta responsividad del SACRI se ha definido en numerosas ocasiones de forma coloquial en el mundo de los OPAC y otros interfaces a sistemas de información como la capacidad de evitar que el usuario se quede "colgado". Aparte de mediante un continuo feedback entorno a los errores y actuación del usuario, la responsividad se consigue también facilitandole una información lo más completa posible sobre lo que está ocurriendo en el sistema, allanando de esta manera sus procesos de toma de decisiones. Esta cualidad suele ser referida también con el término transparencia. Por último, en el mejor de los casos, se espera también que el interfaz sea capaz de proporcionar esta ayuda adaptándose a la situación y características del usuario. Así pues, podemos concluir nuestra definición afirmando que la amigabilidad de un interface se concreta en cualidades específicas como la responsividad (que cuando es una característica continua se transforma en interactividad), en la capacidad de adaptación al nivel del usuario, la creación de un entorno de comunicación "natural" y a la transparencia del sistema.

Desgraciadamente, en muchas ocasiones la amigabilidad del interfaz de usuario va en perjuicio de su rapidez de uso y de su eficacia operativa. Quizá sería necesario desarrollar un nuevo concepto, que podríamos denominar interactividad ecológica, que integrara los conceptos de amigabilidad — en cuanto que adaptabilidad al usuario, y, por tanto, capacidad ergonómica- $\mathrm{y}$ eficacia adaptativa a su entorno funcional cambiante - ecológico. Constituiría una especie de correlato cognitivo y dinámico de lo que el concepto "Ergonomía" supone en el campo del diseño de herramientas físicas.

\section{Medida de la amigabilidad de un interface}

Así, pues, la "facilidad" de uso es un concepto fértil en sugerencias. El problema se plantea cuando llega la hora de evaluar y comparar la amigabilidad de diversos interfaces (LAPOINTE, 1990). Para ello podemos recurrir a dos tipos de medidas: en primer lugar, las medidas de satisfacción subjetiva — ya sea de expertos o nóveles-, por medio de cuestionarios y escalas estandarizadas; y, en segundo lugar, los experimentos y cuasiexperimentos. Las primeras tienen la enorme ventaja de proporcionarnos información sobre el sentir del sujeto, lo cual es relamente importante puesto que estamos intentando ofrecer un producto que satisfaga a personas de una manera global. Sin embargo, estos datos son muchas veces difíciles de interpretar y están sujetos a errores de apreciación. Así, por ejemplo, un interfaz de usuario puede presentar la información de una forma estética y agradable, pero puede prolongar excesivamente los tiempos de obtención de información. Aún peor, la estética puede convertirse en una "cosmética", en un camuflaje o en un intento de seducción del usuario potencial, que oculte

Scire. $1: 1$ (en.-jun. 1995). 
otras características no deseables del sistema.

Sin desechar —-muy al contrario—, la investigación de la satisfacción subjetiva, la investigación de la amigabilidad del interfaz de usuario debe apoyarse también en metodologías científicas de tipo experimental. Es necesario operacionalizar el concepto de "amigabilidad" y explicitarlo por medio de una relación funcional y medible entre una variable independiente y una variable dependiente en el seno de una tarea de recuperación de información. Las variables independientes serán, lógicamente, problemas de decisión relacionados con la manipulación de información automatizada propuestos por el investigador al usuario. La variable dependiente más fácilmente objetivizable es, sin duda, el tiempo de reacción; es decir, la cantidad de tiempo que transcurre entre la presentación del estímulo al sujeto experimental y el comienzo de una respuesta. Este paradigma admite variaciones muy complejas, como la medida del tiempo que pasa entre la presentación de un problema y la consecución de un resultado previamente especificado, el tiempo de ejecución de la misma tarea en diversos sistemas y un largo etcetera. Con ayuda de estos experimentos, es posible medir como influye la configuración estimular del interfaz de usuario en la capacidad de respuesta del usuario. De hecho, y como argumento en pro de la utilidad de este tipo de trabajos, el tiempo de reacción se ha convertido en el paradigma experimental clásico en Psicología Cognitiva, desde que fuera utilizado por Hemlholtz a mediados del siglo pasado para medir la velocidad de conducción del impulso nervioso.

También resultan enormemente fructíferos los paradigmas experimentales típicos de la Psicología del Aprendizaje, en particular los relacionados con el aprendizaje operante. En este caso el lapso entre el estímulo — un problema de recuperación de información - y la respuesta se mide durante una serie de ensayos y se representa por medio de una tasa o un gráfico estandarizados y comparables para distintos grupos de usuarios y entornos de recuperación. De esta manera es posible analizar en qué medida un interfaz dado favorece el rápido aprendizaje de estrategias de recuperación de información (3).

Por supuesto, la utilización de paradigmas experimentales en la investigación sobre interfaces hombre máquina supone un cuidadoso estudio de la situación experimental y de las variables intervinientes — que deben ser controladas-, tales como la constancia del medio, las actitudes, conocimientos y experiencia previos, etc.

\section{Breve historia del interfaz hombre-máquina}

Desde el mismo momento de su concepción, el ordenador digital aparece como una herramienta fundamentalmente matemática, que aprovecha las posibilidades del código binario para domesticar la circulación de energía eléctrica por

Scire. $1: 1$ (en.-jun. 1995). 
una estructura física, haciendo posible la resolución de cualquier problema que pueda ser expresado en una fórmula matemática.

El ser humano corriente, por su parte, utiliza como herramienta de comunicación lo que denominamos 'lenguaje natural' - por contraposición a los lenguajes artificiales, matemáticos o de otro tipo- Sin embargo, el lenguaje natural no ha podido ser recodificado hasta el momento en términos matemáticos, $\mathrm{o}$, si preferimos, no ha podido ser totalmente formalizado a pesar de los importantes avances conseguidos.

No es extraño, por tanto, que desde el primer momento se planteara el problema de la interacción entre el hombre y la computadora (human-computer interface). Se trataba de un problema eminentemente práctico, pero con importantes implicaciones filosóficas insertas dentro del debate sobre la relación entre el hombre y la máquina. Por ello, este debate atrajo el interés no sólo de ingenieros y matemáticos, sino de psicólogos, lingüístas y aún filósofos (4).

En la práctica, cabe detectar una evolución muy clara en el interfaz hombreordenador, desde los complicados sistemas de los albores del ordenador hasta los increibles sistemas de interacción que empezamos a disfrutar en nuestros días.

Al principio, la interacción se realizaba en el propio código binario del ordenador (bits). En seguida se desarrollaron suscesivas agrupaciones heurísticas de bits efectuadas para aproximar el código binario a los códigos decimales y alfabéticos que dominan los sistemas de cuenta y escritura modernos — Binary Coded Decimal System o BCD (sucesivamente de 4, 6 y ocho bits)—. Estos sistemas de codificación permitían ya un procesamiento completo de los significantes de las lenguas naturales - ya que no de sus significados-. La comunicación con el ordenador se basaba en técnicas desarrolladas a finales del siglo XIX en los departamentos de estadística de los gobiernos más avanzados. Los datos se introducían mediante tarjetas o cintas perforadas y se obtenían los resultados impresos por medio de impresoras. Ambas podían ser empleadas en combinación con un teclado. En la superficie del papel perforado, toda la información estaba perfectamente estructurada en campos, normalizada, y transpuesta a códigos numéricos con el objeto de conseguir la máxima parsimonía en la comunicación, y el consiguiente ahorro máximo de tiempo y, por tanto, de dinero en la entrada de datos. Sin embargo, a pesar de ello, presentaba graves inconvenientes: exigía personal absolutamente especializado, los procesos de entrada de datos y verificación requerían una enorme cantidad de tiempo y, desde el punto de vista del operador, las tareas resultaban enormemente repetitivas y consumían demasiada atención.

La asociación al proyecto informático de las tecnologías audio-vídeo provocaron una primera revolución en el interfaz hombre-ordenador. Un primer avan-

Scire. 1 : 1 (en.-jun. 1995). 
ce fue la conexión de un tele-tipo, cuyo obetivo era proporcionar al usuario retroalimentación siempre que lo deseara sobre los procesos que estaban ocurriendo en el interior del ordenador. La verificación de datos resultaba así enormemente simplificada y la capacidad de interacción hombre-máquina provisionalmentemultiplicada. Los tele-tipos fueron pronto desplazados por los monitores de vídeo, creando el nuevo mundo informático en el que nos movemos hoy. Por otra parte, los nuevos sistemas de almacenamiento magnético acortaban de manera radical los tiempos de procesamiento de los datos alamacenados en memorias externas, y, por tanto, aumentaban la velocidad de respuesta del sistema hombremáquina. Estas facilidades de interacción pusieron las condiciones suficientes para la aparición de lenguajes pseudo-naturales a principio de los años cincuenta encargados de facilitar el envío de órdenes de propósito general a la máquina y de proporcionar el necesario feed-back por parte del ordenador: los sistemas operativos y los lenguajes de programación de alto nivel. Por supuesto, se trataba de lenguajes artificales por su alta normalización, y por la profusión de recursos lingüísticos exclusivamente matemáticos. Sin embargo, preferimos denominarlos lenguajes pseudo-naturales, mejor que artificiales, por dos razones: a) porque semejaban en gran manera un lenguaje natural simplificado, limitado a las necesidades concretas de comunicación del sistema informático, y reducido fundamentalmente al modo de comunicación imperativo (5); y b) porque su objetivo era facilitar al usuario la interacción con la máquina. Efectivamente, aunque pueda parecer mentira a mediados de los noventa, tenían en cuenta sus necesidades cognoscitivas, puesto que se acercaban por medio de abreviaturas a expresiones de significado semejante en la vida corriente; en inglés, por supuesto. Desde el punto de vista del usuario, la interacción con el ordenador se realizaba mediante órdenes imperativas con una serie de parámetros opcionales que modificaban el alcance de la orden dentro de un universo de posibilidades cerrado. Para conseguir que el ordenador ejecutase un determinado conjunto de instrucciones, las órdenes se introducían en una línea de órdenes seguidas del mandato de ejecución (RETORNO o ENTRAR), o se almacenaban en los ficheros de ejecución diferida (ficheros ejecutables o programas). El conjunto de las órdenes disponibles en el sistema configuraba un auténtico lenguaje de ordenes -lenguaje artificial, pero lenguaje al fin y al cabo-; cuya simplificada gramática consistía en la formulación de la orden, seguida de la especificación de los parámetros, o variables que especifican su ejecución (6). Sin duda, se trataba de un sistema de interacción hombre/máquina mucho más próximo al lenguaje natural que los anteriores sistemas, pero seguía requiriendo un personal de alto nivel intelectual y con una capacidad de abstracción muy notable (7).

De cara a la entrada de datos era necesario desarrollar interfaces más sencillos para su uso por personal sin conocimientos en lenguajes de órdenes. Para ello

Scire. $1: 1$ (en.-jun. 1995). 
se empezaron a ensayar soluciones ya existentes con anterioridad a la informática. Una de las primeras, muy apta para construir y gestionar bases de datos, fue implementar en la pantalla formularios, combinando etiquetas y espacios para la introducción de datos. La pantalla se convertía así en una unidad de comunicación compuesta de elementos relacionados en función de un objetivo: representar entidades del mundo real y las operaciones o tareas que es necesario efectuar sobre esas representaciones. Así, por ejemplo, un programa de gestión de bibliotecas requerirá sendas pantallas para cada una de las operaciones bibliotecarias básicas: adquisición, registro, préstamo, recuperación de información etc. En cada una de estas pantallas dispondremos de los programas pertinentes de manipulación de datos — por ejemplo, eliminación y creación de registros bibliográficos, expurgo, transferencia a otros módulos, etc.—, cuya clave será normalmente una tecla de función u otra combinación inequívoca de teclas. La función de préstamo, por su parte, puede analizarse en una serie de tareas individuales gestión de prestatarios, préstamo, renovación, devolución, etc.- - que dará lugar a las correspondientes pantallas. Este es el sistema de desarrollo de interfaz de usuario que podemos denominar diseño función-pantalla, en el que se analizan y anidan jerarquicamente las tareas y funciones que se deben realizar, y se representan en cuantas pantallas sean necesarias. La función de la pantalla es presentar conjuntamente toda la información necesaria para tomar decisiones de cara a la realización de una actividad bien definida, así como una serie de llamadas a las herramientas disponibles para manipular esa información y ejecutar las actividades necesarias. La ejecución de las acciones programadas, tradicionalmente llamada mediante instrucciones, se realiza ahora pulsando una tecla o una combinación de teclas (frecuentemente las de función combinadas con otras teclas no alfanuméricas), siguiendo sugerencias especificadas en la propia pantalla. El personal que utiliza los programas por medio de interfaces de este tipo debe ser necesariamente especializado, pero no tiene por qué alcanzar los estándares del programador. Se iniciaba la era del operador informático.

No obstante el avance realizado, la combinación de un gran número de pantallas en un mismo programa podía provocar una gran despiste en el operador. Para resolver este problema se recurrió de nuevo a viejas soluciones, en concreto, al que en los libros se denomina tabla de sumarios o en un restaurante menú: un inventario de opciones disponibles entre las que cabe realizar una elección. Algunas de estas pantallas se convertían en índices que incluían un grupo de pantallas relacionadas, y que permitían seleccionar una de ellas por medio de las teclas de movimiento y retorno, las de función, un ratón o el recurso a una pantalla táctil. Vendrán luego sofisticaciones como los cuadros de ayuda, o los pulldown menús, capaces de aumentar enormente el panel de órdenes ofrecido en una sóla pantalla. De la mano de todos estos avances, el mundo de la pantalla se 
va haciendo enormemente complejo.

Como podemos ver, las opciones analizadas hasta el momento dependían radicalmente del código escrito. En seguida, los ingenieros informáticos empezaron a perseguir los logros de sus avezados colegas dedicados a la televisión y concentraron su esfuerzo en la conquista de la imagen. La incorporación de la imagen y el sonido resultó revolucionaria al añadir al ordenador una dimensión auténticamente ecológica: consiguió acercar la informática a los modos de comunicación y canales de información del hombre corriente. El interfaz de usuario se convierte a través de la pantalla de vídeo, el altavoz y el micrófono en una metáfora de la realidad e incorpora una enorme cantidad de imágenes de uso cotidiano: el dedo, el puntero, el botón, el escritorio, la puerta, el cajón, etc. De esta forma, el inasequible y abstracto mundo de la información electrónica se objetiviza y se convierte en un mundo accesible al común de los mortales. La importancia de esta auténtica revolución todavía no se percibe con claridad, pero no en vano se ha hablado del CD-Interactivo como el nuevo papiro. Es necesario prestar en este punto un homenaje a los denostados pioneros de esta frontera, conquistada en primer lugar por los juegos de ordenador, el primer producto informático que pudo ser autenticamente considerado de consumo de masas.

Por fin, los informáticos y sus aliados de otras ciencias se han lanzado a la conquista de lo que actualmente parece la última frontera del interfaz hombremáquina: el dominio del lenguaje natural y la profundización en la realidad virtual. La conquista del lenguaje va acompañada de esfuerzos semejantes en la dilucidación y formalización del pensamiento (Sistemas expertos). Los rápidos avances en traducción automática, sistemas expertos, servidores vocales y otros campos hacen presagiar una victoria casi absoluta en un plazo no muy lejano.

En realidad, los distintos sistemas de diseño del interfaz de usuario poseen ventajas e inconvenientes bien contrastados, y ello explica que frecuentemente convivan en un mismo programa. Por ejemplo, un sistema de información puede poseer un interfaz gráfico para usuarios no expertos, muy intuitivo y fácil de manejar, y puede poseer también un lenguaje de órdenes que permita busquedas y operaciones más complejas y rápidas a un usuario avezado a través de la línea de órdenes o ficheros ejecutables. Los diferentes interfaces se pueden desplegar a lo largo de un continuum que parte de la máxima parsimonía, formalización, precisión rapidez de uso y necesidad de entrenamiento y conocimientos previos, hasta la máxima facilidad de uso y amigabilidad. Como hemos señalado anteriormente, el objetivo final es la conciliación y alianza de ambos contrarios.

\section{Fundamentos cognitivos del interfaz amigable}

El desarrollo de interfaces amigables está firmemente apoyado en la investi-

Scire. $1: 1$ (en.-jun. 1995). 
gación básica y aplicada realizada por los psicólogos del aprendizaje y los psicólogos cognitivos. La Psicología Cognitiva se ha desarrollado como un paradigma alternativo al conductista: se interesa por los fenómenos no directamente accesibles a la observación directa que ocurren en el interior de esa "caja negra" que es la mente humana. Aunque plantea un nuevo marco teórico y ensancha el espectro de problemas de la Psicología, el paradigma cognitivo no renuncia a los mejores logros del conductismo: la psicología experimental, y el modelo EstímuloRespuesta (E/R). El modelo E/R es, como comentabamos en el apartado dedicado a la "medida de la amigabilidad de un interface", especialmente útil para guiar la investigación aplicada sobre interfaces. La Psicología Cognitiva se encuentra, además, estrechamente relacionada con la Informática, hasta el punto de que ambas ciencias comparten una misma visión de los sistemas inteligentes como procesadores de información, y ambas ciencias se han visto mutuamente influidas y han intercambiado ideas e investigadores con cierta frecuencia. El hombre es visto a través de su artefacto más depurado — la computadora—, y es interpretado analógicamente a partir de lo que se ha venido denominando la metáfora del ordenador.

Ciertamente, resulta difícil negar que las personas recibimos, procesamos, almacenamos y respondemos información. Todo nuestro sistema nervioso constituye un extraordinario mecanismo capaz de reducir la complejidad del ambiente, representársela y responder a ella conforme a esas representaciones. Efectivamente, la información se percibe en forma de conjuntos de estímulos organizados, de configuraciones o estructuras de estímulos. A lo largo de nuestra evolución biológica y cultural como organismos sociales, hemos ido desarrollando mecanismos adaptativos que condicionan nuestra manera de captar la realidad. Si no clasificaramos y organizaramos la información que llega a nuestro organismo seríamos rápidamente desbordados por ella, y nos veríamos privados de la capacidad de actuar. La necesidad de agrupar la información de cara a facilitar su procesamiento ha sido ilustrada teóricamente y demostrada experimentalmente por varios psicólogos cognitivos. Broadbent $(1958,1971)$ propuso el modelo básico que ha guiado a los investigadores posteriores. Según dicho modelo el sistema nervioso se asemeja a un canal de comunicación de capacidad limitada, y está compuesto por los sentidos — que captan y procesan la información en paralelo y la guardan durante milésimas de segundo en una memoria sensorial-, un buffer de almacenamiento temporal donde se almacena la información ya categorizada — la memoria a corto plazo o primaria—, y por un filtro que selecciona la información; finalmente, la información ya procesada puede ser depositada en una memoria a largo plazo o secundaria. Este modelo sería depurado y enriquecido por Atkinson y Shriffin (1968), que estudiaron variables tales como el tipo de procesamiento, tasa de olvido, etc., que acontece en cada una de

Scire. $1: 1$ (en.-jun. 1995). 
las memorias mencionadas.

En el contexto de la investigación sobre el procesamiento humano de la información se han hecho gran cantidad de observaciones de gran interés para el diseño de interfaces. Así, por ejemplo, Miller (1956) demostró que la limitación de la memoria de trabajo humana — la memoria a corto plazo — rondaba los siete items; $y$, lo que es más importante, que la estrategia que la mente utilizaba para procesar conjuntos más complejos era integrar los elementos en grupos (en inglés, chunks). La jerarquía parece ser más eficaz a la hora de facilitar el recuerdo basado en agrupamiento que la sóla asociación (COFER, 1965), lo que refuerza la teoría de Miller sobre la creación de estructuras de orden superior. Todas estas realidades constituyen el fundamento cognitivo de técnicas como el menú de opciones, los menús de ventana (рор-ир menus), los menús desplegables o "de persiana" (pull-down menus), etc. Se desprende también de manera lógica de esta evidencia experimental que un interface tiene que estar más organizado cuanto más cantidad de información pretenda proporcionar. El tipo de organización debe ser conceptual y acercarse más a la organización mental del usuario que a la del diseñador.

Uno de los resultados más consolidados en la experimentación sobre la memoria es que a las personas nos resulta más rápido reconocer que recordar (por ejemplo, Mandler, Pearlstone y Koopmans, 1969). Los buenos interfaces ofrecen todos los punteros necesarios a la memoria del usuario para facilitarle el reconocimiento de las acciones que debe solicitar a la máquina. En caso de duda, ofrecen ayudas locales y generales que amplían esos punteros.

También ha sido firmemente probada la conveniencia de que los interfaces faciliten la generalización, proceso por el cual aprendizajes realizados en una situación dada se transfieren a otras situaciones análogas (Domjan y Burkhard, 1990). Esto ha sido fundamentado desde la teoría del aprendizaje y la teoría de la memoria. Los entornos estables y con grandes semejanzas para distintos tipos de actividades facilitan enormemente el aprendizaje y la consolidación de la memoria. Aumentan los ensayos de aprendizaje, evitan el decaimiento y disminuyen el efecto de la interferencia de señales competidoras en la consolidación de la huella mnésica. Este objetivo de diseño se debe aplicar no sólo a las instrucciones, sino también a las estrategias globales de actuación.

La generalización explica también el auge de los entornos metafóricos y orientados a objetos. Los entornos familiares facilitan la inferencia comunicativa y el reaprovechamiento de habilidades ya aprendidas. Esto, unido a que respondemos más rápido a señales discretas (8) que a señales articuladas (iconos frente a palabras), justifica el éxito imparable de los entornos icónicos tipo Macintosh, Windows y X-Windows.

Scire. 1 : 1 (en.-jun. 1995). 
En resumen, podemos afirmar que el procesador humano de información se debate entre dos polos opuestos: el hambre de información y la necesidad de estabilidad. La atención pasiva es captada por la diferencia, la atención activa es dirigida por procesos guiados a un objetivo. Como dirían los psicólogos de la Gestalt, ambos tipos de atención necesitan distinguir adecuadamente entre fondo y forma; ambas son un compromiso entre la diferencia y la uniformidad. Desde este paradigma, la comunicación hombre/máquina surge como un compromiso entre, por un lado, la potencia, cualidad distintiva y la organización de los estímulos del interfaz y, por el otro, la atención puesta por el usuario, que, sea pasiva o dirigida, estará siempre condicionada por sus experiencias previas, estrategias consolidadas y las leyes de la percepción, la memoria y el aprendizaje. Entre ambos sistemas se produce una interacción basada en la inferencia comunicativa, que puede ser auxiliada y optimizada. La inevitable experiencia de las distancias entre el usuario concreto y el usuario ideal de un sistema sugiere el desarrollo de puentes que amplien el número de personas que acceden a los servicios de información automatizada y, paralelamente, el mercado para los productos de información.

\section{Técnicas de la amigabilidad}

Todos los aspectos teóricos que hemos ido reflejando en las páginas anteriores sugieren el empleo de una serie de técnicas que han sido utilizadas con éxito en la ingeniería de sistemas de información:

- Combinación de códigos comunicativos distintos: visual (icónico), verbal, auditivo. El objetivo es conseguir una redundacia óptima en el proceso de comunicación para empalmar los enfasis y tiempos de respuesta, aumentando además la potencia de la señal.

- Organización y estructuración del interface en niveles jerárquicos para superar las limitaciones de la memoria de trabajo y del espacio paerceptivo (pulldown menus, pop-up menus, estructuración gráfica, etc.)

- Propiciar la inferencia metafórica en el proceso comunicativo mediante las posibilidades de los entornos gráficos: v. g. Macintosh, Windows. Las posibilidades de representación e interacción icónica de estos sistemas permite emplear símbolos usuales en la vida cotidiana de los usuarios. Otra estrategia alternativa es realizar un estudio exhaustivo de los conceptos implicados en un dominio dado, y proceder sistemáticamente a su representación icónica.

- Procurar ayudas textuales y gráficas, tanto generales como locales.

- Procurar ayudas procedimentales, simulando el asesoramiento de un experto, ya sea mediante procedimientos encubiertos o trasparentes al usuario.

Vamos a ver a continuación algunas experiencias en el campo de la informa-

Scire. $1: 1$ (en.-jun. 1995). 
ción bibliográfica que pueden mostrarnos la utilidad de estas estrategias de comunicación hombre/máquina o, por lo menos, líneas de investigación a seguir.

\section{Algunas experiencias en el campo de la información bibliográfi- ca}

El tema de los interfaces de búsqueda bibliográfica — los famosos OPAC (Open Public Access Catalogues) — ha sido objeto de numerosos estudios, tanto de caracter general (Belkin, 1991; Fugmann, 1991; Hildreth, 1987; 1989a; 1989b; 1991; Larson, 1992), como limitados al entorno europeo (Jones, 1989), el norteamericano (Hildreth, 1987; Gerhan, 1989) o dedicados a estudiar el estado de la cuestión en distintos paises eurpeos como el Reino Unido (Greenwood, 1989; Slack, 1989; Wood, 1989; BAT, 1990), Francia (Jouguelet, 1989) o Alemania (Schnelling, 1988). Vamos a revisar a continuación algunos proyectos punteros, para los cuales existen resultados consolidados, y que ejemplifican algunas de las técnicas de amigabilidad más importantes que hemos repasado (9).

El proceso seguido en el desarrollo de los distintos interfaces es semejante. En una primera fase se analizan de forma exhaustiva las estrategias de búsqueda de los usuarios; se evalúa su satisfacción e interacción con otros sistemas comerciales; y se les solicita información sobre sus necesidades y posibilidades de satisfacción. En una segunda fase se modeliza toda la información obtenida conceptualmente; y, seguidamente, se construye un prototipo. Por fin, se evalúa el desempeño y la satisfacción de los usuarios con el nuevo sistema, comparándolo con los datos extraídos para los otros sistemas respecto a los que pretende innovar.

6.1. Sistemas con ayuda local intensiva: el ejemplo de Icarus.

En 1991 F. J. Murphy, A. S. Pollitt y P. R. White (1991) presentaron el resultado de un proyecto de investigación HCI (Human Computer Interaction) desarrollado por la School of Computing and Mathematics y la Biblioteca del Polytechnic of Huddersfield con una subvención del British Library Research \& Development Department. El objetivo era desarrollar un interfaz para OPAC centrado en el usuario denominado ICARUS, dotado de posibilidades gráficas, y fundamentarlo previamente en un exhaustivo estudio de las estrategias comunmente seguidas por los usuarios, y compararlo con el OPAC del sistema BLCMP. Los resultados se consideraron positivos. Lo característico de ICARUS era un interfaz muy interactivo de tipo gráfico basado en el uso del ratón sobre botones que etiquetaban los campos de búsqueda disponibles para los distintos tipos de información bibliográfica, y cuya pulsación provoca su consiguiente apertura. A la izquierda de la pantalla aparece otro campo destinado a proporcionar ayudas locales de forma intensiva, conforme a los botones pulsados. Paralelamente el

Scire. 1 : 1 (en.-jun. 1995). 
sistema envía información en ventanas de ayuda conforme encuentra los problemas tipificados para cada estrategia de búsqueda (10). El sistema asiste además al usuario en la consulta de los listados de autoridades, incluidas las materias.

\subsection{Entornos metafóricos: The Book House e IRMA.}

The Book House es un proyecto de investigación danés desarrollado por Annelise Mark Pejtersen y sus colaboradores (Agger y Jensen, 1989; Goodstein y Pejtersen; Pejtersen, 1989a, 1989b, 1990), cuyo informe final fue publicado en 1989, y que fue presentado como propuesta al Plan de Actuación para Bibliotecas del Programa Telemático de la CE en 1991 con el nombre de IRMA (Information Retrieval with Metaphor-based Access). The Book House es un proyecto que integra un interfaz de usuario gráfico, absolutamente icónico, y un sistema de clasificación facetado para literatura, bajo el paraguas teórico de un modelo cognitivo de las necesidades del lector de este tipo de documentos y de sus estrategias de satisfacción.

La clasificación facetada es el resultado de diversas investigaciones de campo realizadas en los años setenta y consta de cuatro grandes facetas: a) la intención del autor - experiencia emocional, información, educación, efectos que persigue en el lector-; b) el contexto - lugar y tiempo, entorno geográfico, histórico, social o profesional—; c) el tema — trama, interacciones psicológicas, relaciones sociales—; y d) la accesibilidad — características físicas del libro, legibilidad, características de la impresión, formato, editor.

Las estrategias principales utilizadas por el usuario son la búsqueda analítica —un típico ejemplo de estrategia orientada a la solución de problemas-, la búsqueda analógica —el usuario busca algo semejante a otro libro que le gustó-, y la búsqueda no dirigida —el simple hojeo de las estanterías.

El interfaz gráfico se basa en el principio de que los datos y los signos son procesados con más facilidad si están fuertemente organizados, especialmente si esta organización es predecible por el usuario. De forma semejante a la conocida metáfora del escritorio - fundamento cognitivo del interfaz del Macintosh y de Windows-, la base de datos se simboliza por medio de una casa llena de libros, por la cual el usuario debe desplazarse hasta encontrar lo que busca. Las estrategias de búsqueda están representadas por sendas pantallas que semejan habitaciones, dentro de las cuales el usuario dispone de diversas herramientas representadas icónicamente. Por ejemplo, en la habitación destinada a la búsqueda analítica, el usuario dispone de iconos que representan, entre otros útiles, a los operadores booleanos y otro conjunto de iconos que representan los distintos temas disponibles. En la habitación donde se opera la búsqueda analógica, el usuario puede ojear los índices de autores y temas para facilitarle la identificación de su libro de referencia, el cual tiene un peso asignado en virtud de los términos

Scire. 1 : 1 (en.-jun. 1995). 
que han servido para clasificarlo; seguidamente el sistema intenta localizar otros ejemplares semejantes, y, si existen, los muestra por orden decreciente de relevancia.

En suma, The Book House constituye un interfaz extraordinariamente innovador por su firme aparato teórico, y por el original acercamiento que toma para resolver el problema del usuario inexperto que se inicia en la búsqueda bibliográfica de literatura de ficción.

6.3. Sistemas expertos que auxilian la búsqueda: el proyecto Okapi.

El objetivo básico de la recuperación documental es, como sabemos, su optimización. Ello se consigue maximizando la obtención de registros relevantes y minimizando, a la vez, el número de registros irrelevantes (ruido). La búsqueda depende de las habilidades y estrategias del usuario, el entorno físico y tecnológico, la estructura de los datos, el sistema de representación de los conceptos (lenguajes de indización y clasificación) y el lenguaje de recuperación (García Marco, 1991)

Stephen Walker y sus colaboradores se han concentrado precisamente en el problema de auxiliar al usuario en su búsqueda de registros de manera que éste pueda obtener fácilmente al menos un registro relevante; y ello al menos por tres razones: a) muchos usuarios se desaniman si deben esforzarse para localizar información; b) otros usuarios creen que el ordenador les está dando una respuesta definitiva y que la biblioteca no tiene nada que les pueda ser útil; c) la mayor parte de las búsquedas en bibliotecas no especializadas quedan satisfechas por un sólo hallazgo (11); d) a partir del ejemplar recuperado, el sistema puede intentar encontrar otros semejantes (Jones, 1988). De manera inversa, el sistema ahorrará mucho tiempo al usuario si es capaz de informarle rápidamente de que no existe en la colección nada que pueda sarisfacer sus necesidades. La investigación realizada se ha ido plasmando en un prototipo de acceso público en línea denominado Okapi (Mitev, Venner y Walker, 1985; Walker, 1988; Walker, 1989). Para conseguir estos objetivos el sistema incorpora una serie de herramientas encubiertas que funcionan independientemente de la voluntad del usuario.

La ayuda encubierta debería empezar por minimizar los errores del propio usuario, en partircular, proporcionándole información sobre posibles errores ortográficos, incluida la puntuación (Walker y Jones, 1987). A continuación, el sistema comprueba automáticamente la existencia de sinónimos y cuasi-sinónimos en la base de datos bibliográfica, y trunca las raices si es necesario. ${ }^{12} \mathrm{El}$ tratamiento de los términos va seguido de una fórmula de conexión de los términos diferente de la normalmente usada, denominada best match keyword searching, y que produce una ecuación de búsqueda diferente. En la mayoría de los siste-

Scire. 1 : 1 (en.-jun. 1995). 
mas, los términos a buscar son conectados por defecto mediante operadores de intersección implicitos. Por el contrario, Okapi, en vez de buscar los registros que contienen todos los términos introducidos, asigna un peso a cada término que es inversamente proporcional a la frecuencia del término en la base de datos, y muestra en pantalla todos los registros cuyos términos proporcionan una suma ponderada que sobrepasa un umbral previamente establecido, ordenados según esa suma (13).

Por fin, el sistema permite al usuario ampliar la búsqueda de diversas maneras: a) invitando al usuario a consultar documentos que poseen el mismo número de clasificación, materias, autor o serie; y b) ejecutando una expansión de búsqueda automática en base a las palabras clave contenidas en los registros considerados relevantes (14). El procedimiento de expansión automática parece proporcionar excelentes resultados.

\section{Conclusiones}

Evidentemente, los sistemas de información son el objetivo prioritario de ese área punta de desarrollo de la informática que es el interfaz hombre máquina. Su objetivo último y declarado es facilitar la comunicación de los hombres con esas nuevas memorias auxiliares externas y activas - frente a la pasividad del documento- que son, en último término, los ordenadores. Para ello, se debe profundizar en las diversas facetas de esa comunicación: a) las características de ambas partes en cuanto que procesadores de información; b) las características específicas de esos procesos de transferencia de información que acontecen entre el usuario y la computadora ; y c) las posibilidades de desarrollo de las capacidades existentes de ambos sistemas de información (hombre y máquina), y, en el caso del sistema automatizado, su eventual rediseño. Los sistemas de información bibliográfica son, potencialmente, una de las más interesantes plataformas de investigación en el campo del interfaz hombre-máquina . Y ello por varias razones: por su creciente generalización, su evidente rentabilidad social, la extendida percepción sobre las limitaciones informativas de los sistemas comerciales ante distintos grupos de usuarios y por la existencia de una amplia comunidad científica interesada por el tema.

Sin embargo, se trata de un área de investigación especialmente compleja, caracterizada precisamente por su desbordante mutabilidad. Precisamente ahora que empezabamos a alcanzar en nuestro país un cierto dominio de las nuevas técnicas de interacción hombre/ordenador, corremos de nuevo el riesgo de quedar de nuevo ahogados por la ola del cambio tecnológico. Recientemente, Ben Sshneiderman, de la University of Maryland, con motivo del anuncio de una conferencia internacional, profetizaba en la Internet la llegada de la siguiente generación de interfaces gráficos de usuario, caracterizados por un ingreso sin com-

Scire. 1 : 1 (en.-jun. 1995). 
plejos en el mundo de la realidad virtual :

The wildly popular graphical user interfaces (GUIs) are an improvement over command languages, but the next generation of user interfaces is already on the way. The aging GUIs with clumsy one-window-at-a-time housekeeping will give way to rapid, coordinated multiple windows. The future will be dynamic, spatial, 3dimensional, virtual, ubiquitous, gestural, colorful, often auditory, and sometimes immersive. The demand for high resolution multimedia and full-motion video will push the hardware requirements, absorb network capacity, and challenge the algorithm designers. How can we balance the desire for innovation with the need for stability?

Las posibilidades futuras del desarrollo de interfaces de información bibliográfica son, pues, enormes, y combinarán dos grandes líneas: a) el desarrollo de mecanismos sensoriales de interacción cada vez más próximos a la realidad cotidiana del usuario; y b) la implementación de sistemas de ayuda, tanto interactivos como ocultos- que pondrán a su alcance los conocimientos y estrategias del experto.

\section{Notas}

(1) Ingwersen (1991) ha desarrollado una innovadora y completa visión del proceso de información documental desde una perspectiva cognitiva.

(2) Empleamos aquí el concepto de SACRI de forma genérica, sin referirnos a un sistema en particular o asilado. De hecho, una de las grandes ventajas de la concepción del interfaz como un elemento autónomo del sistema de información es que resulta posible diseñar un interfaz que actúe de ventana a sistemas muy diferentes, proporcionando al usuario un entorno único (gateway). Así , por ejemplo, en febrero de 1992 BRS/SEARCH anunuciaba su nuevo interfaz de usuario DISNET (Domain Independent Intelligent Information and Services Network Interface)—, capaz de asegurar al acceso a diferentes bases de datos, independientemente del lenguaje de recuperación concreto de cada una de ellas, y proporcionando ayuda local y general intensiva de modo que el usuario pueda optimizar su uso de las distintas bases minimizando la necesidad de ayuda experta.

(3) Véase el reciente trabajo de Belén Altuna (1992) sobre los comportamientos de uso y estregias de búsqueda de los usuarios de catálogos automatizados.

(4) Véase Cummins y Pollock (1992).

(5) En cierta manera, cabría afirmar que los lenguajes de programación de alto nivel son, más que lenguajes plenamente artificiales, subconjuntos altamente normalizados de los lenguajes naturales con un propósito muy específico: controlar sistemas informáticos. Esta proximidad funcional entre los lenguajes operativos y de programación de alto nivel a los naturales está permitiendo el desarrollo de lenguajes de programación enfocados a usuarios inexpertos muy semejantes al inglés normal.

(6) A la unión de estos elementos la denominaremos a partir de ahora instrucción.

(7) En realidad, la comunicación hombre/máquina por medio de lenguajes de órdenes es,

Scire. 1 : 1 (en.-jun. 1995). 
aunque compleja, la más versátil y potente hasta el momento.

(8) Sobre la cualidad distintiva del significante que desata el proceso de recuerdo se han realizado numerosas investigaciones — por ejemplo, entorno al tipo de símbolos o al uso del color - que pueden consultarse en la bibliografía adjunta.

(9) No vamos a estudiar ningún sistema funcionando en lenguaje natural, aunque se han realizado prototipos aplicados a la recuperación bibliográfica. Así, por ejemplo, el sistema francés ALEXIS fue diseñado para batirse con preguntas en lenguaje cotidiano (Jouguelet, 1989).

(10)Por ejemplo, si se solicita una búsqueda por autor, el sistema pide al usuario que especifique si se trata de un autor personal o una entidad.

(11)Efectivamente, las búsquedas exhaustivas se suelen realizar en colecciones y bases de datos especializadas.

(12)Estas estrategias configura el punto de partida de una base de conocimientos léxicos — a la que Walter denomina "hyper-thesauri"—, incluyendo relaciones jerárquicas, relaciones asociativas y homónimos, que sería efectiva también para la ampliación de búsquedas y para disminuir la tasa de ruido causada por los homónimos.

(13)Walters proporciona cifras que comparan este tipo de estrategia de búsqueda con el "y" implícito: entre un 10 y un $20 \%$ de búsquedas dejan de ser nulas, y de un 30 a un $50 \%$ de búsquedas proporcionan más registros relevantes.

(14)La ecuación que proporciona el peso de cada término $(\mathrm{Pt})$ es la siguiente: $\mathrm{Pt}=\log [(\mathrm{r}$ / $(\mathrm{R}-\mathrm{r})) /((\mathrm{n}-\mathrm{r}) /(\mathrm{N}-\mathrm{n}-\mathrm{R}+\mathrm{r}))$ ]; donde $\mathrm{N}$ es el número de registros de la base de datos, $\mathrm{n}$ es el número de registros que contienen el término, $\mathrm{R}$ es el número de registros relevantes, y r es el número de registros relevantes que contienen el término.

\section{Bibliografía}

Agger, S.; Jensen, H. (1989). The Book House : visual design. Copenhagen : Royal Academy of Fine Arts, School of Architecture, 1989.

Alltuna Esteibar, Belen. (1992). Comportamientos de uso y estregias de búsqueda de los usuarios de catálogos automatizados : breve revisión de la investigación. // Federación Española de Sociedades de Archivística, Biblioteconomía y Documentación. Miscelánea-homenaje a Luis García Ejarque. Madrid : FESABID, 1992. p. 103-111.

American Society for Information Science. Mid-Year Meeting (1989. San Diego, Calif.). The user inteface: 1989 ASIS Mid-year Meeting, May 21-24, 1989, San Diego, California : abstracts of papers. Washington, D.C. : American Society for Information Science, 1989.

American Society for Information Science. Mid-Year Meeting (5th. 1976. Vanderbilt University). Information interaction: compedium of presentations of the 5th ASIS Mid-Year Meeting, Vanderbilt University, Nashville, Tennessee, May 20-22, 1976. Washington, D. C.: American Society for Information Science, 1976.

Atkinson, R. C.; Shiffrin, R. M. (1968). Human memory : a proposed system and its control processes. En: Spence, K. W.; Spence, J. T. (eds.). The Psychology of learning and motivation : advances in research and theory. New York : Academic Press, 1968.

Scire. 1 : 1 (en.-jun. 1995). 
Vol. 2, p. 89-195.

Authoring systems. Lister Hill monograph. LHNCBC 92-1. [Bethesda, MD]: U.S. Dept. of Health and Human Services, Public Health Service, National Institutes of Health, National Library of Medicine, 1992.

Bat, Chris (1990). The cutting edge (II). // Public Library Journal. 5 : 4 (1990) 101-103.

Belkin, N. J. User interfaces for information systems. // Journal of Information Science. 17 (1991) 327-344.

Borenstein, Nathaniel S. (1991). Programming as if people mattered : friendly programs, software engineering, and other noble delusions. Princeton, N.J.: Princeton University Press, 1991.

Broadbent, D. E. (1958). Perception and communication. New York : Pergamon Press, 1958

Broadbent, D. E. (1971). Decision and stress. London : Academic Press, 1971.

California. University. Library Systems Development Program. Library system definition: functions \& interfaces. Santa Barbara, Calif.: [s.n.], 1971.

Canadian Workplace Automation Research Centre. Organizational Research Directorate (1990). User-friendliness of interfaces: proposal for an evaluation methodology. Laval, Quebec: Canadian Workplace Automation Research Centre, Organizational Research Directorate, 1990.

Clinic on Library Applications of Data Processing (23rd. 1986. University of Illinois at Urbana-Champaign). What is user friendly?. [Urbana, Ill.] : Graduate School of Library and Information Science, University of Illinois at Urbana-Champaign, 1987.

Clinic on Library Applications of Data Processing (25th. 1988. University of Illinois at Urbana-Champaign). Design and evaluation of computer/human interfaces: issues for librarians and information scientists. [Urbana, Ill.] : Graduate School of Library and Information Science, University of Illinois at Urbana-Champaign, 1991.

Cofer, C. N (1965). On some factors in the organizational characteristics of free recall. En: American Psychologist. N. 20 (1965); p. 261-272.

Crawford, Walt (1986). Patron Access Project, phase I. Development issues. Stanford, Calif.: Research Libraries Group, Research \& Development Division, 1986.

Cummins, Robert ; Pollock, John (1992). Philosophy and AI: Essays at the Interface. Cambridge (Mass.) : MIT, 1992.

Domjan, Michael ; Burkhard, Barbara (1990). Principios de parendizaje y de conducta. Madrid : Debate, 1990.

Efthimiadis, Efthimis Nikolaos (1990). A review of online searching aids. [London]: British Library, Research and Development Dept., 1990. (British Library research paper ; 86).

Fels, S. Sidney (1990). Building adaptive interfaces with neural networks the glove-talk pilot study. [microform]. Ottawa: National Library of Canada, 1990.

Fugmann, Robert (ed.) (1991). Tools for Knowledge Organization and the Human Interface. Frankfurt/Main : Indeks Verlag, 1991. 2 vols.

García Marco, Francisco Javier. De la consulta de catálogos a la gestión de información:

Scire. 1 : 1 (en.-jun. 1995). 
Tensiones hacia el cambio en el diseño de OPACs. // Boletín de la ANABAD. 41 : 34 (jul.-dic. 1991) 325-334.

Gateway software and natural language interfaces : options for online searching. Ann Arbor, Mich.: Pierian Press, 1988. (Library hi tech special studies series; no. 2).

Gerhan, David R. (1989). LCSH in vivo : subject searching performance and strategy in the OPAC era. // Journal of Academic Librarianship. 15 : 2 (May 1989) 83-89.

Goodstein, L. P.; Pejtersen, A. M. (1989). The Book House : system for functionality and evaluation. Ris $\emptyset$ National Laboratory, 1989.

Greenwood, Derek. (1989). OPAC research in the UK. // Journal of Librarianship. $21: 3$ (July 1989) 157-173.

Hildreth, Charles R. (1987a). Beyond boolean : designing the next generation of online catalogues. // Library Trends. 35 (Spring 1987) 647-667.

Hildreth, Charles (1987b). Library Automation in North America: A Reassessment of the impact of new technologies on networking. Munich : K.G. Saur, 1987.

Hildreth, Charles R. (ed.) (1989a). The Online Catalogue : Developments and directions. London : The Library Association, 1989.

Hildreth, Charles (1989b). Intellligent interfaces and retrieval methods for subject searching in bibliographic retrieval systems. Washington : Library of Congress, Cataloging Distribution Service, 1989. (Advances in Library Information Techonology ; 2).

Hildreth, Charles R. (1991). End users and structured searching of online catalogues: Recent research findings. // Fugmann, Robert (ed.). Tools for Knowledge Organization and the Human Interface. Frankfurt/Main : Indeks Verlag, 1991. 2 vols. Vol. 2, p. 9-24.

Ho, Raymond Kim (1988). CHILDS a library environment for children. [microform]. Ottawa: National Library of Canada, 1988.

Ingwersen, Peter (1991). Intermediary functions in information retrieval interaction. Copenhagen : Faculty of Business Administration, Institute of Informatics and Management Accounting, Copenhagen Susiness School, 1991.

Interfaces for information retrieval and online systems: the state of the art. New York: Greenwood Press, 1991.

Jones, Richard M. (1988). A comparative evaluation of two online public access catalogues : user opinions about the design of online catalogues. London : British Library, 1988.

Jouguelet, Suzanne (1989). Subject access in online catalogs : developments in France. // Cataloging \& Classification. 10 : 1-2 (1989) 213-224.

Larson, Roy (1992). Evaluation of advanced retrieval techniques in an experimental online catalog. // Journal of the American Society for Information Science. 43 : 1 (1992) 34-53.

Lapointe, Francois (1990). User-friendliness of interfaces : proposal for an evaluation methodology. Laval, Quebec: Communications Canada, Canadian Workplace Automation Research Centre, Organizational Research Directorate, 1990. 
Local interfaces to the Australian Bibliographic Network : a study. [Canberra]: National Library of Australia and Australian Advisory Council on Bibliographical Services, 1982 (1983 printing). (Development of resource sharing networks : networks study ; 21).

Mandler, G. ; Pearlstone, Z. ; Koopmanx, H. S. (1968). Effects of organization and semantic similarity in a recall and recognition task. // Journal of verbal learning and verbal behaviour. 8 (1968) 410-423.

Miller, G. A. (1956). The magical number seven, plus minus two : some limits of our capacity for processing information. // Psychological Review. 63 (1956) 81-97.

Mitev, Natalie Nadia ; Venner Gillian M. ; Walker, Stephen (1985). Designing an online public access catalogue : OKAPI, a catalogue on a local network. London : Britisn Library, 1985. (British Library Researcgh Report ; 39).

Mitev, Natalie Nadia; Walker, Stephen (1985). Information retrieval aids in an online public access catalogue : automatic intelligen search sequencing. // Informatics 8 : advances in intelligent retrieval : proceedings of an Aslib/BDS conference : Oxford, 16-17 April 1985. London : Aslib, 1985.

Murphy, F. J.; Pollitt, A. S.; White; P. R. (1991). Matching OPAC User Interfaces to User Needs. London : British Library Research \& Development Department, 1991. (British Library R \& D Report ; 6041).

Myers, Brad A. (1988). Creating user interfaces by demonstration. [microform]. Ottawa: National Library of Canada, 1988.

Pejtersen, Annelise Mark (1989a). The Book house : modelling users' needs and search strategies as a basis for system design. Ris $\emptyset$ National Laboratory, November 1989.

Pejtersen, , Annelise Mark (1989b). A library system for information retrieval based on a cognitive task analysis and supported by an icon-based interface. // ACM, SIGIR Conference Proceedings. Boston, 1989. p. 40-47.

Pejtersen, Annelise Mark (1990). Icons for representation of Domain Knowledge in Interfaces. Paper presented at the ISKO Conference in Darmstadt, 1990. Darmstadt, 1990.

Pilote, Michel (1983). A framework for the design of linguistic user interfaces. [Ottawa: National Library of Canada], 1983.

Richard M. (1989). Online catalogue research in Europe. // Journal of the American Society for Information Science. $40: 3$ (May 1989) 153-157.

Schnelling, Heiner (1988). OPAC design : a European perspective. // Dempsey, Lorcan (ed.). Influencing the system designer: online public access to library files : proceedings of the Third National Conference held at Bath University, England, 12-15 September 1987. Oxford : Elsevier Advanced Technology Publications, 1988. p. 8798.

Slack, Fran (1988). Subject searching on OPACs : a general survey of facilities available on OPACs in academic libraries in the UK. // Vine. 72 (Nov. 1988) 8-15.

Walker, Stephen ; Jones, Richard M. (1987). Improving subject retrieval in online catalogues : 1 : stemming, automatic spelling correction and cross-reference tables. London : British Library, 1987. (Library Research Report ; 39).

Scire. 1 : 1 (en.-jun. 1995). 
Walker, Stephen (1988) . OKAPI : developing an intelligent interactive online catalogue. // Vine. 71 (1988).

Walker, Stephen (1989). The Okapi online catalogue research projects. // Hildreth, Charles R. The online catalogue : developments and directions. London : Library Association, 1989. p. 84-106.

Walker, Stephen ; De Vere, Racheld (1990). Improving subject retrieval in online catalogues : 2 : relevance feedback and query expansion. London : British Library, 1990. (British Library Research Paper ; 72).

Wood, Anthony J. (1989). OPACs in public libraries : a second look. // Public Library Journal. 4 : 4 (JulyAug. 1989) 75, 77, 79, 81.

Scire. $1: 1$ (en.-jun. 1995). 